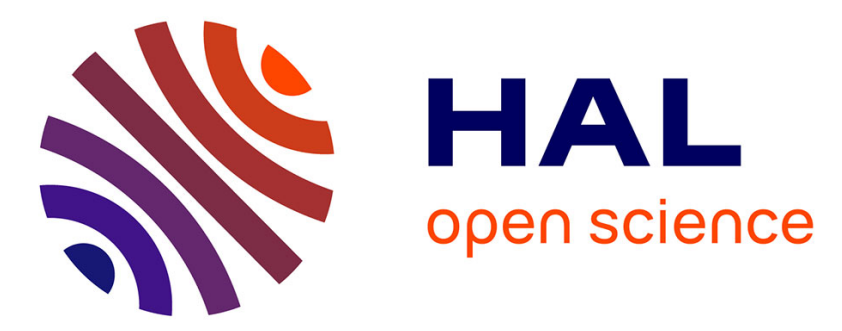

\title{
Field-Free Magnetization Switching by an Acoustic Wave
}

\author{
I.S. S Camara, J.-Y. Duquesne, A. Lemaître, C. Gourdon, L. Thevenard
}

\section{To cite this version:}

I.S. S Camara, J.-Y. Duquesne, A. Lemaître, C. Gourdon, L. Thevenard. Field-Free Magnetization Switching by an Acoustic Wave. Physical Review Applied, 2019, 11 (1), 10.1103/PhysRevApplied.11.014045 . hal-02187172

\section{HAL Id: hal-02187172 \\ https://hal.sorbonne-universite.fr/hal-02187172}

Submitted on 17 Jul 2019

HAL is a multi-disciplinary open access archive for the deposit and dissemination of scientific research documents, whether they are published or not. The documents may come from teaching and research institutions in France or abroad, or from public or private research centers.
L'archive ouverte pluridisciplinaire HAL, est destinée au dépôt et à la diffusion de documents scientifiques de niveau recherche, publiés ou non, émanant des établissements d'enseignement et de recherche français ou étrangers, des laboratoires publics ou privés. 


\title{
Field-free magnetization switching by an acoustic wave
}

\author{
I. S. Camara, ${ }^{1}$ J.-Y. Duquesne, ${ }^{1}$ A. Lemaître ${ }^{2}$ C. Gourdon,${ }^{1}$ and L. Thevenard ${ }^{1, *}$ \\ ${ }^{1}$ Sorbonne Université, CNRS, Institut des Nanosciences de Paris, 4 place Jussieu,75252 Paris France \\ ${ }^{2}$ Centre de Nanosciences et de Nanotechnologies, CNRS, \\ Univ. Paris-Sud, Université Paris-Saclay, 91460 Marcoussis, France
}

(Dated: July 16, 2019)

\begin{abstract}
Surface acoustic waves (SAW) propagating on magneto-strictive ferromagnets can induce magnetization reversal: their weak damping and mature technology make them ideal for remote wave control of magnetic bits. Experimental demonstrations of this spectacular coupling have so far required the simultaneous application of a static magnetic field. We show here SAW-driven allacoustical switching (AAS) over millimetric distances. It relies on the triggering of magnetization precession of a uniaxial in-plane magnetized film by acoustic ferromagnetic resonance at zero field. Moreover, the absence of biasing field enables toggling between the two equilibrium magnetic states for over twenty consecutive acoustic pulses. This proof-of-concept opens an alternative path to magnetic bit manipulation.
\end{abstract}

\section{INTRODUCTION}

Switching magnetic bits without the requirement for magnetic fields has been a constant quest in the spintronics and nanomagnetism communities, as it would enable a denser packing and easier addressing of magnetic bits in logic and memory devices, with limited crosstalk. This search has been rewarded by the discovery of all-optical[1], electric field- and current-induced magnetization switching. For the latter two however[2-7], the necessity for biasing fields remains ubiquitous, whether spin-transfer, spin-orbit torques, or electric-field modulations of the anisotropy are at play. A recently developed alternative for magnetization switching is the use of acoustic waves [8-15]. The effective field generated by Rayleigh waves through inverse magnetostriction can switch magnetization efficiently in magnetostrictive thin films of both in-plane and out-of-plane anisotropies $[14,15]$. In these references, the mechanism was ascribed to resonant switching [8, 9]: the SAW triggers a highly non-linear, large amplitude magnetization rotation around a bias (resonance) field chosen to match the magnetic precession with the acoustic frequencies typically 0.1-1 GHz. One way to eliminate the need for this field is to engineer a system where the precession frequency matches the acoustic frequency at zero field. This is the key ingredient of our results: the thermal dependence of magnetic parameters in (Ga,Mn)As, a dilute magnetic semiconductor used as a demonstrator to achieve zero-magnetic field SAW switching at a well chosen temperature. Moreover, we demonstrate toggling between the two equilibrium magnetic states by over twenty consecutive acoustic pulses, opening the way to a fieldfree wave-control of magnetization.

\section{SAMPLE}

The experiments are performed on a 45-nm-thick layer of $\left(\mathrm{Ga}_{0.95}, \mathrm{Mn}_{0.05}\right) \mathrm{As}$ grown by molecular beam epitaxy on a non-intentionally doped GaAs (001) substrate. The Curie temperature $T_{C}$ reaches $120 \mathrm{~K}$ after a $16 \mathrm{~h} / 200^{\circ} \mathrm{C}$ anneal. The layer has a strong in-plane uniaxial anisotropy along [1ํㅣ $]$ up to $T_{C}(1 \mathrm{mT}$ coercive field at $T=100 \mathrm{~K})$. Please refer to Ref. 15 for more details on this sample. A $2 \times 2 \mathrm{~mm}^{2}$ magnetic mesa is defined by wet etching [Fig. 1(a)]. To excite and detect surface acoustic Rayleigh waves, $60 \mathrm{~nm}$-thick $\mathrm{Al}$ interdigitated transducers (IDTs) are deposited on either side of the mesa by lift-off. The "split-44" transducer design [16] was used: 15 pairs of 4 equi-potential and equi-spaced digits that can be excited by a radio-frequency (rf) voltage. With a nominal base periodicity of $\lambda=20 \mu \mathrm{m}$, SAWs could be

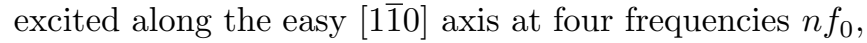
with $n=1,3,5,7$ and $f_{0}=V_{r} / \lambda=141 \mathrm{MHz}$ where $V_{r}$ is the Rayleigh wave velocity. Here we will only present work at $7 f_{0}=990 \mathrm{MHz}$, as it was shown to be the one maximizing the switching efficiency [15].

\section{ZERO-FIELD SAW-DRIVEN SWITCHING}

To evaluate the effect of a single SAW burst on the layer magnetization we performed longitudinal Kerr microscopy (632-nm LED illumination, NA=0.4 objective). The sample magnetization was initialized as $+\mathbf{M}_{0} \|[1 \overline{1} 0]$ with a permanent magnet, and a reference image acquired close to the emitting transducer (dashed contour in Fig. 1(a)). A single 250-ns-long rf pulse (990 MHz) was then applied to the IDT using a Keysight N5172B generator, an image taken and divided by the reference one in order to show in normalized contrast the switched areas. A field-regulated CAYLAR coil ensured that the external field was $B=0 \pm 0.1 \mathrm{mT}$ at the sample location. Since we expect the resonance fields to decrease with increasing 


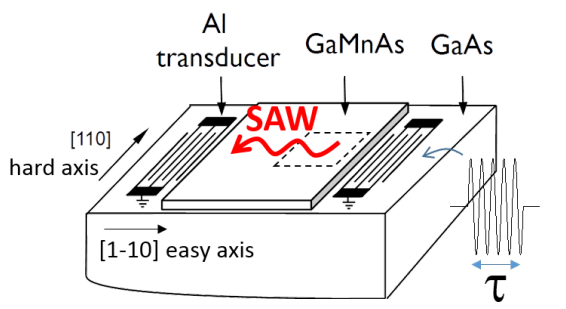

(a)

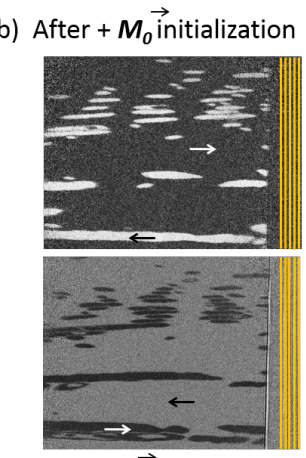

(c) After $-\overrightarrow{M_{0}}$ initialization

FIG. 1. (a) Schematics of the set-up. For the switching experiments, Kerr microscopy images are taken over the area delimited by the dashed contour. For SAW-FMR experiments (Fig. 3), $\tau$-long pulses are applied to the right transducer. The field is applied along the hard axis [110] and the opposite transducer detects the acoustic echo. (b,c) Kerr microscopy differential images $\left(306 \times 410 \mu \mathrm{m}^{2}\right)$ after a single $250 \mathrm{~ns}-\mathrm{long}$ SAW burst at $T=100 \mathrm{~K}\left(B=0 \mathrm{mT}, P_{r f}=31 \mathrm{dBm}\right)$, and opposite initializations. The black and white arrows indicate the magnetization direction.

temperature, the temperature was set to $T=100 \mathrm{~K}$, sufficiently close to the Curie temperature to expect low-field resonance with the SAW, as will be shown in Sec. IV, but far enough to maintain a saturation magnetization of $20 \mathrm{kA} \mathrm{m}^{-1}$.

Fig. 1(b) shows that after the SAW pulse, the magnetization has switched in certain locations (white domains). No field-free switching is observed at low rf power, or when the transducer is excited out of its resonance bandwidth (e.g. at $980 \mathrm{MHz}$ ), ruling out an influence of heating or of the electromagnetic rf radiation from the IDTs. Elongated oblong domains are formed, a few tens of micron wide, and up to $300 \mu \mathrm{m}$-long. Similar shapes were observed after resonant switching under field at $20 \mathrm{~K}$ [15]. This is explained as follows. At both of these temperatures, the SAW makes the magnetization oscillate between one equilibrium position and the other as soon as the absolute value of the strain reaches a certain threshold. When the rf excitation is stopped, the final magnetic configuration depends critically on the sign of the strain as it drops down to zero and crosses once more the threshold [8, 9, 14]. Micromagnetic simulations clearly show that a small dispersion of the sample magneto-elastic coefficients makes the SAW-switching unequally efficient across the layer, since the threshold depends on the detuning between the local precession frequency and the SAW frequency, and on the strain amplitude [15]. This leads to the formation of extended patches of switched magnetization, in patterns that vary with the combination of $P_{r f}$ and $f_{\text {saw }}$ that is used (as evidenced by comparing Figs. 1(b), 2(a) and 4). Finally, note that the fairly long burst length used for switching is imposed by

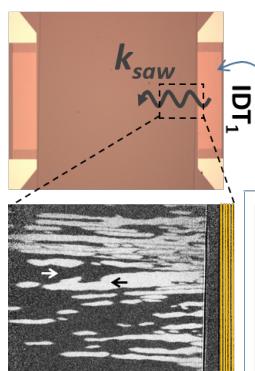

(a) $+32 \mathrm{dBm}$

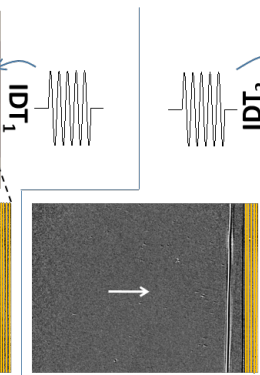

(b) $+32 \mathrm{dBm}$

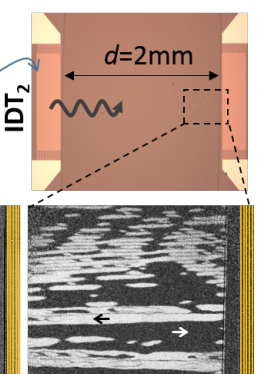

(c) $+35 \mathrm{dBm}$
FIG. 2. Zero-field SAW-driven magnetization switching following a 250-ns-long 987-MHz rf pulse applied to the right (a) or left $(\mathrm{b}, \mathrm{c})$ transducer (images $\left.306 \times 410 \mu \mathrm{m}^{2}\right)$. When observing far from the excitation, acoustic and magneto-acoustic losses need to be compensated to obtain a similar switching efficiency, hence the larger power $(+35 \mathrm{dBm})$ needed for excitation through $\mathrm{IDT}_{2}$.

the long acoustic risetimes of our device (90 ns), the IDT having been designed for narrow bandwidth multiple harmonics excitation, rather than short transients.

Initializing $-\mathbf{M}_{0}$ this time and applying the same SAW burst shows that switching also occurs (black domains in Fig. 1(c)), and that some areas reverse regardless of the initial saturation, $-\mathbf{M}_{0}$ or $+\mathbf{M}_{0}[23]$. This is in stark contrast with the resonant switching observed under field at $20 \mathrm{~K}$ : minute variations of the field alignment with the hard axis across the sample prevent some areas to switch with the same efficiency from $-\mathbf{M}_{0}$ or $+\mathbf{M}_{0}$ initial configurations [15]. A similar favoring of one magnetization configuration over another was also routinely seen in the early days of current-driven precessional spin-valve switching [4], an influence of the polarizing layer dipolar field. An efficient solution had been the use of a synthetic antiferromagnet as polarizer to limit the influence of biasing fields on the free layer [17]. Here, suppressing the need for a field by working at higher temperature proves to be a fruitful strategy to balance $-\mathbf{M}_{0} \rightarrow+\mathbf{M}_{0}$ and $+\mathbf{M}_{0} \rightarrow-\mathbf{M}_{0}$ switching probabilities.

Let us underline that the likeliness between switched patterns starting from $+\mathbf{M}_{0}$ or $-\mathbf{M}_{0}$ also rules out that switching is due to a spurious static field along the easy axis, where domain nucleation could be favored by the SAW: it would then only be efficient starting from one configuration. Contrary to what we believe to be a resonant switching route here, this would be a non-resonant SAW-assisted mechanism, which has already been evidenced in $(\mathrm{Ga}, \mathrm{Mn})(\mathrm{As}, \mathrm{P}), \mathrm{Co} / \mathrm{Pt}$ and FeGa $[10,11,13]$.

To evaluate up to which distance from the exciting transducer this effect remains efficient, we took two magneto-optical images close to the right transducer $\mathrm{IDT}_{1}$ : after applying an $\mathrm{rf}$ pulse either to $\mathrm{IDT}_{1}$ [Fig. 2(a)], or to the opposite one, IDT $2,2 \mathrm{~mm}$ away [Figs. 2(b,c)]. Switching remains efficient provided that the applied power is increased by $+3 \mathrm{~dB}$ in the latter case. 


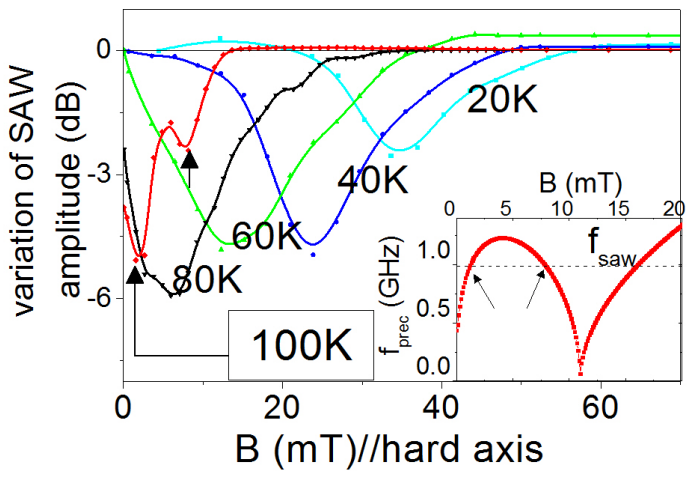

FIG. 3. Relative transmitted SAW amplitude versus hard axis field, at high rf power. Inset: Field dependence of the $T=100 \mathrm{~K}$ precession frequency calculated taking into account the anisotropy constants determined by cavity FMR. In both graphs, the arrows point to the resonance conditions for which the precession frequency most closely matches $f_{S A W}=990 \mathrm{MHz}$.

This compensates for the SAW magneto-acoustic attenuation (see further the $T=100 \mathrm{~K}$ SAW-FMR curve in Fig. 3), and to a lesser degree for the native attenuation of the SAW in GaAs[22], and for the weak asymmetry of the IDT transduction efficiencies. A similar long-distance efficiency had been shown when using the SAW to reduce transiently domain nucleation barriers [13]. This demonstrates once more the relevance of SAWs for remote switching.

\section{ZERO-FIELD SAW-DRIVEN FERROMAGNETIC RESONANCE}

To assess whether resonant SAW-induced magnetization precession is indeed the mechanism responsible for this zero-field SAW-driven reversal, we now present SAW-driven ferromagnetic resonance experiments (SAWFMR). Obtaining this resonance is a pre-requisite for magnetization switching $[14,15]$. Here the layer is magnetically initialized $+\mathbf{M}_{0}$ along the easy axis [110] , and $400 \mathrm{~ns}-$ long rf bursts at $1 \mathrm{kHz}$ repetition rate are delivered to $\mathrm{IDT}_{1}$. The transmitted SAW amplitude and phase variations are measured on the echo detected at $\mathrm{IDT}_{2}$ as the in-plane field is increased [Fig. 1(a)]. With the applied field along the hard axis, the magnetization is progressively oriented away from the easy axis, leading to a decrease of the magnetization precession frequency (inset of Fig. 3). The magnetization is mainly coupled to the SAW longitudinal strain via the anisotropic magnetoelastic coupling coefficient commonly called $B_{2}$ in metallic ferromagnets [18]. The transverse strain intervenes to a lesser degree, via weaker higher-order magnetoelastic coupling coefficients [15].

Figure 3 shows the SAW amplitude variations for
$T=20-100 \mathrm{~K}$, at $f_{\mathrm{SAW}}=990 \mathrm{MHz}$ at high SAW power $\left(P_{r f} \approx 35 \mathrm{dBm}\right)$. At all temperatures a resonant absorption occurs at the field for which the mean precession frequency most closely matches $f_{\text {SAW }}[15,19,20]$, a clear signature of SAW-induced magnetization precession. The resonant field decreases from $35 \mathrm{mT}$ at $20 \mathrm{~K}$ to about $1.5 \mathrm{mT}$ at $100 \mathrm{~K}$. At this temperature, a second resonance peak is present at higher field, $8 \mathrm{mT}$, where the precession frequency crosses $f_{\mathrm{SAW}}$ a second time (inset of Fig. 3).

Let us now discuss the choice of temperature and $\mathrm{rf}$ power for field-free switching experiments. At sufficiently low SAW power (not shown), the transmitted SAW amplitude varies linearly with the incoming one and the precession amplitude is small, as evidenced experimentally, and proportional to the strain amplitude [21]. At zero field there is no absorption and no torque on the magnetization either $[19,20]$. At the typical powers and temperature used here however $(+31-35 \mathrm{dBm}, \mathrm{T}=100 \mathrm{~K})$ the SAW transmission becomes non linear and the resonance shifts down to lower field, with the SAW-FMR absorption remaining finite at zero field (Fig. 3). The SAW-induced magnetization dynamics is expected to become very nonlinear with complex trajectories [9], a pre-requisite for precessional switching $[9,14]$. A certain level of absorption must be reached to observe field-free switching however: none was seen at $80 \mathrm{~K}$ (at the maximum available rf power) where there is a weaker zero-field SAW attenuation than at $100 \mathrm{~K}$.

\section{ZERO-FIELD SAW-DRIVEN MAGNETIZATION TOGGLING}

In the macrospin vision of precessional switching, the final magnetic configuration depends on the compressive or tensile nature of the strain as it decays down to zero at the end of the burst and crosses the switching threshold $[8,9,14]$. For consecutive identical rf bursts, it should thus be possible to toggle the magnetization between $-\mathbf{M}_{0}$ and $+\mathbf{M}_{0}$ with high fidelity, a determinism critical when manipulating magnetically encoded information. At $T=20 \mathrm{~K}$, such reversibility cannot be obtained: applying successive pulses barely modifies the switched magnetization pattern, because even minute misalignments of the bias field with the static local magnetization robustly favor one configuration over the other [15].

In order to test whether magnetization could be switched locally and repeatedly between $\pm \mathbf{M}_{0}$ by an acoustic wave, without field this time, we proceed as follows. At $\mathrm{T}=100 \mathrm{~K}$ a reference image is taken after a $+\mathbf{M}_{0}$ initialization, and 30 consecutive rf bursts of nominally identical phase and duration are applied at $10 \mathrm{~s}$ intervals, taking a Kerr image after each one. At the end of the series, another reference image is taken after saturating $-\mathbf{M}_{0}$. A spatial analysis of the local intensity $I(\mathbf{r})$ 

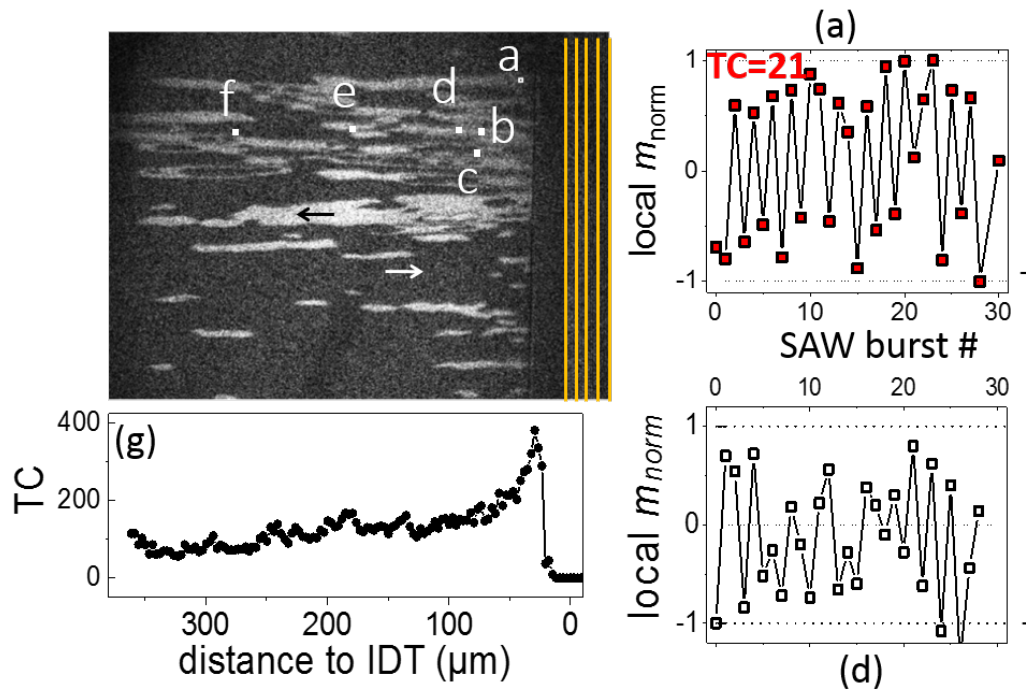

(d) (b)
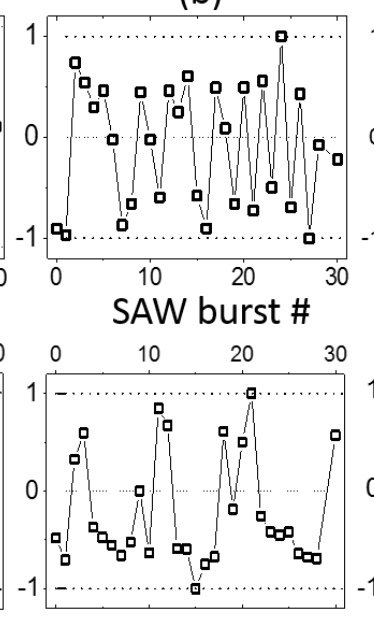

(e) (c)
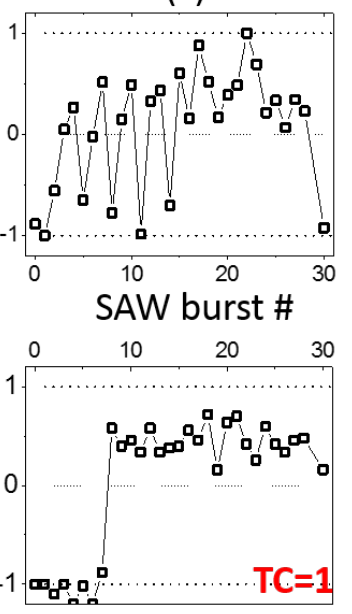

(f)

FIG. 4. (a-f) Zero-field toggling behavior under successive $250 \mathrm{~ns}-$ long $990 \mathrm{MHz}$ SAW bursts at $T=100 \mathrm{~K}(B=0 \mathrm{mT}$, $P_{r f}=35 \mathrm{dBm}$, image $\left.306 \times 410 \mu \mathrm{m}^{2}\right)$, starting from a $+\mathbf{M}_{0}$ configuration: normalized local magnetization of selected $10 \times 10$ pix ${ }^{2}$ bins. Labels on the image locate the position of each bin with respect to the IDT. (g) Toggling count (TC, defined in [23]) summed over the SAW wavefront, as a function of the distance to the SAW emitter.

of these images is performed numerically to obtain the local normalized magnetization of the $n$-th burst image: $m_{\text {norm }}^{n}(\mathbf{r})=2 \frac{I^{n}(\mathbf{r})-I_{+\mathrm{M}_{0}}(\mathbf{r})}{I_{-\mathrm{M}_{0}}(\mathbf{r})-I_{+} \mathrm{M}_{0}(\mathbf{r})}-1$. This value between -1 and +1 corresponds to the average normalized magnetization of a $2.9 \times 2.9 \mu \mathrm{m}^{2}$ area $\left(10 \times 10 \mathrm{pix}^{2} \mathrm{bin}\right)$ centered around r. Figure 4a-f shows the switching behavior for a few representative bins. Bin a is located about $36 \mu \mathrm{m}$ from the exciting IDT, and excellent toggling is observed between $-\mathbf{M}_{0}$ and $+\mathbf{M}_{0}$ states. As the distance to the exciting transducer increases (bins $\mathbf{b}, \mathbf{c}$ at $\approx 70 \mu \mathrm{m}, \mathbf{d}$ at $\approx 80 \mu \mathrm{m}$ and e at $170 \mu \mathrm{m}$ ), this behavior becomes overall less robust. Bin $\mathbf{f}$ shows only one switching event in the $30 \mathrm{SAW}$-pulses series, and does not switch back to its initial state unless the sample is resaturated. Instead, consecutive SAW bursts result in slow domain expansion, a compelling phenomenon that will be developed in an upcoming communication. A similar phenomenology was observed when starting from the $-\mathbf{M}_{0}$ uniform configuration.

We define the toggling count, TC, as the number of large-contrast jumps in the 30-burst series (see [23] for details). While some pockets of high TCs can be found both close to the transducer and far from it (see SM), vertically averaging the toggling efficiency shows a clear overall degradation when going away from the IDT (Fig. $4 \mathrm{~g})$. To explain this in the framework of precessional switching, the acoustic field seen by a given location should be significantly modified from burst to burst, for instance by spatial and temporal phase fluctuations of the SAW wavefront, which would induce decoherence in the resonant switching process. Variations of the Rayleigh wave velocity (due to a temperature drift, or modification of the sample surface with time) can be safely discarded as a likely explanation since it would only delay the arrival of the SAW burst, but not influence its end phase, i.e. the strain sign as it crosses the switching threshold. Instead, an incoherent scattering of the wave as it travels on the multi-magnetic domain layer can be invoked. The numerous magnetic domain-walls it must cross may modify its phase and amplitude, acting as a scattering landscape varying after each burst. Finally, competing mechanisms might also contribute to stochastic non-resonant reversals, such as the aforementioned transient decrease of domain nucleation barriers, or domain-wall motion/creation.

\section{CONCLUSIONS}

We have presented a proof-of-concept demonstration of SAW-driven all-acoustical switching. Working on the dilute magnetic semiconductor $(\mathrm{Ga}, \mathrm{Mn}) \mathrm{As}$, we relied on the steep decrease of the resonance field matching precession and SAW frequency with increasing temperature to reverse magnetization over millimetric distances. Deterministic two-way toggling was evidenced in several spots, but the overall efficiency was shown to decrease with the distance to the transducer, a possible consequence of the multi-domain configuration due to the dispersion of the magnetic parameters and large sample size. The optimal configuration for zero-field SAW switching should therefore rely on a room-temperature ferro- or ferri-magnet exhibiting a combination of low precession frequency 
yet high magnetostriction, structured into monodomain structures, and SAWs excited by an impedance-matched IDT centered around a few GHz. Complemented by wave-front shaping or focusing IDTs, this will enable to select spatially a magnetic structure using acoustics.

\section{ACKNOWLEDGEMENTS}

This work has been partly supported by the French Agence Nationale de la Recherche (ANR13-JS04-000101) and the French RENATECH network. We acknowledge M. Bernard (Institut des Nanosciences de Paris) for technical assistance.

* thevenard@insp.jussieu.fr

[1] S. Mangin et al., Engineered materials for all-optical helicity-dependent magnetic switching, Nature Materials 13, 286 (2014)

[2] W. K. Hiebert, L. Lagae, and J. De Boeck, Spatially inhomogeneous ultrafast precessional magnetization reversal, Phys. Rev. B 68, 020402 (2003).

[3] I. N. Krivorotov, Time-Domain Measurements of Nanomagnet Dynamics Driven by Spin-Transfer Torques, Science 307, 228 (2005).

[4] C. Papusoi, B. Delaet, B. Rodmacq, D. Houssameddine, J.-P. Michel, U. Ebels, R. C. Sousa, L. D. BudaPrejbeanu, and B. Dieny, 100 ps precessional spintransfer switching of a planar magnetic random access memory cell with perpendicular spin polarizer, App. Phys. Rev. 95, 072506 (2009).

[5] D. Bedau, H. Liu, J.-J. Bouzaglou, A. D. Kent, J. Z. Sun, J. A. Katine, E. E. Fullerton, and S. Mangin, Ultrafast spin-transfer switching in spin valve nanopillars with perpendicular anisotropy, App. Phys. Rev. 96, 022514 (2010).

[6] Y. Shiota, T. Nozaki, F. Bonell, S. Murakami, T. Shinjo, and Y. Suzuki, Induction of coherent magnetization switching in a few atomic layers of FeCo using voltage pulses, Nature Materials 11, 39 (2011).

[7] H. Zhang, Z. Hou, J. Zhang, Z. Zhang, and Y. Liu, Precession frequency and fast switching dependence on the in-plane and out-of-plane dual spin-torque polarizers, App. Phys. Rev. 100, 142409 (2012).

[8] O. Kovalenko, T. Pezeril, and V. V. Temnov, New Concept for Magnetization Switching by Ultrafast Acoustic Pulses, Phys. Rev. Lett. 110, 266602 (2013).

[9] L. Thevenard, J.-Y. Duquesne, E. Peronne, H. J. von Bardeleben, H. Jaffrès, S. Ruttala, J.-M. George, A. Lemaître, and C. Gourdon, Irreversible magnetization switching using surface acoustic waves, Phys. Rev. B 87, 144402 (2013).

[10] W. Li, B. Buford, A. Jander, and P. Dhagat, Acoustically Assisted Magnetic Recording: A New Paradigm in Magnetic Data Storage, IEEE Trans. Mag. 50, 3100704 (2014).
[11] U. Singh and S. Adenwalla, Spatial mapping of focused surface acoustic waves in the investigation of high frequency strain induced changes, Nanotechnology 26, 255707 (2015).

[12] S. Davis, J. A. Borchers, B. B. Maranville, and S. Adenwalla, Fast strain wave induced magnetization changes in long cobalt bars: Domain motion versus coherent rotation, J. Appl. Phys. 117, 063904 (2015).

[13] L. Thevenard, I. S. Camara, J.-Y. Prieur, P. Rovillain, A. Lemaître, C. Gourdon, and J.-Y. Duquesne, Strong reduction of the coercivity by a surface acoustic wave in an out-of-plane magnetized epilayer, Phys. Rev. B 93, 140405 (2016).

[14] L. Thevenard, I. S. Camara, S. Majrab, M. Bernard, P. Rovillain, A. Lemaître, C. Gourdon, and J.-Y. Duquesne, Precessional magnetization switching by a surface acoustic wave, Phys. Rev. B 93, 134430 (2016).

[15] P. Kuszewski, I. S. Camara, N. Biarrotte, L. Becerra, J. von Bardeleben, W. Savero Torres, A. Lemaître, C. Gourdon, J.-Y. Duquesne, and L. Thevenard, Resonant magneto-acoustic switching: influence of Rayleigh wave frequency and wavevector, J. Phys.: Cond. Mat. 30, 244003 (2018).

[16] F. J. R. Schülein, E. Zallo, P. Atkinson, O. G. Schmidt, R. Trotta, A. Rastelli, A. Wixforth, and H. J. Krenner Fourier synthesis of radiofrequency nanomechanical pulses with different shapes, Nature Nanotechnology 10, 512 (2015).

[17] A. Vaysset, C. Papusoi, L. D. Buda-Prejbeanu, S. Bandiera, M. Marins de Castro, Y. Dahmane, J.-C. Toussaint, U. Ebels, S. Auffret, R. Sousa, L. Vila, and B. Dieny, Improved coherence of ultrafast spin-transferdriven precessional switching with synthetic antiferromagnet perpendicular polarizer, App. Phys. Rev. 98, 242511 (2011).

[18] A. Hubert and R. Schäfer, Magnetic domains, Springer ed. (Berlin, 2000).

[19] L. Dreher, M. Weiler, M. Pernpeintner, H. Huebl, R. Gross, M. Brandt, and S. Goennenwein, Surface acoustic wave driven ferromagnetic resonance in nickel thin films: Theory and experiment, Phys. Rev. B 86, 134415 (2012).

[20] L. Thevenard, C. Gourdon, J.-Y. Prieur, H. J. von Bardeleben, S. Vincent, L. Becerra, L. Largeau, and J.-Y. Duquesne, Surface-acoustic-wave-driven ferromagnetic resonance in $(\mathrm{Ga}, \mathrm{Mn})(\mathrm{As}, \mathrm{P})$ epilayers, Phys. Rev. B 90, 094401 (2014).

[21] P. Kuszewski, J.-Y. Duquesne, L. Becerra, A. Lemaître, S. Vincent, S. Majrab, F. Margaillan, C. Gourdon, and L. Thevenard, Optical probing of Rayleigh wave driven magneto-acoustic resonance, Phys. Rev. Applied 10, 1 (2018).

[22] A. J. Slobodnik, GaAs acoustic-surface-wave propagation losses at $1 \mathrm{GHz}$, Elec. Lett. 8, 307 (1972).

[23] See Supplemental Material at [URL will be inserted by publisher] for details on the effect of the initial saturation and the definition of the toggling count. 